\title{
Outcome for Australian children with acute leukaemia is influenced by ethnicity and the metro-regional divide
}

\author{
Sophie Jessop ${ }^{1}$, Sandra Ruhayel ${ }^{2}$, Danny Youlden ${ }^{3}$, Cynthia Lu $^{3}$, Suzanne Milne ${ }^{1}$, Michelle \\ Henderson $^{4}$, Joanne Aitken ${ }^{3}$, Rosemary Sutton ${ }^{5}$, Rishi Kotecha ${ }^{2}$, and Tamaz Revesz ${ }^{1}$ \\ ${ }^{1}$ Women's and Children's Hospital Adelaide \\ ${ }^{2}$ Perth Children's Hospital \\ ${ }^{3}$ Cancer Council Queensland \\ ${ }^{4}$ Children's Cancer Institute \\ ${ }^{5}$ Children's Cancer Institute, School of Women's and Children's Health
}

September 11, 2020

\begin{abstract}
Objective: To compare disease presentation and outcome in Aboriginal and non-Aboriginal children with acute leukaemia and to assess the impact of remoteness and area-based socioeconomic disadvantage. Design: A retrospective review of children treated for acute leukaemia from South Australia (SA), Northern Territory (NT) and Western Australia (WA) between 2009 and 2018. Setting: Women's and Children's Hospital (WCH), Adelaide and Perth Children's Hospital (PCH) - the sole referral sites for paediatric cancer for SA, NT and WA. Participants: Eligible patients, aged between 1 day and $<18$ years, diagnosed with acute leukaemia. Main Outcome Measures: Leukaemia diagnosis and overall survival. Results: Analysis of 455 children treated for acute leukaemia showed inferior survival outcomes were associated with remote/very remote localities ( $\mathrm{p}=0.004)$. Five-year overall survival was 91.7\% (95\% CI: 87.9\%-94.3\%) for children with ALL and 69.8\% (56.7\%-79.5\%) for AML. A large percentage of Aboriginal children from SA/NT were diagnosed with AML compared to others (60.0\% vs. 14.4\%, p=0.001). A trend towards inferior overall survival was seen for Aboriginal children with ALL compared to non-Aboriginal children (82.4\% vs. $92.2 \%, \mathrm{p}=0.07)$ and $55.6 \%$ were high-risk by study criteria; however, MRD testing did not identify any high MRD cases. Aboriginal children were less likely to be enrolled on clinical trials $(34.5 \%$ vs. $53.1 \%, \mathrm{p}=0.03)$ and more likely to be lost to follow-up ( $41.4 \%$ vs. $13.2 \%, \mathrm{p}<0.001)$. Conclusion: Aboriginal ethnicity and geographic remoteness of residence are adverse prognostic factors for Australian children with leukaemia. Additional strategies are required to ensure improvements in follow-up and survival of these children.
\end{abstract}

\section{INTRODUCTION}

Globally, there is documented variation regarding presenting features, biology and outcome for childhood leukaemia according to different ethnic, geographical and socioeconomic groups ${ }^{1-8}$. Little is known about the impact of ethnicity and geographical distribution on survival rates for childhood leukaemia in Australia. Previous observational studies in South Australia (SA) conducted over successive periods identified a trend for more complex leukaemia with greater treatment resistance among Aboriginal children and in children from remote areas ${ }^{1,2}$, while an Australian registry reported significantly lower five-year overall survival among Aboriginal compared to non-Aboriginal children ${ }^{3}$.

Epidemiological studies have shown the likelihood of survival following childhood cancer diagnosis generally decreases the further an individual lives from a major population centre and in areas with greater socioeconomic disadvantage ${ }^{9}$. Contributing factors are yet to be identified, but may be linked to reduced access to treatment and late detection ${ }^{9}$. 
The purpose of this study was to compare disease presentation and outcome for acute leukaemia in Aboriginal and non-Aboriginal children and to assess the impact of remoteness of residence and area-based socioeconomic disadvantage in SA, Northern Territory (NT) and Western Australia (WA).

\section{METHODS}

\section{Patients and Study Design}

We performed a retrospective review of presenting features and outcomes of all children aged between 1 day and $<18$ years who were diagnosed with acute leukaemia between January 1st 2009 and December 31st 2018 at the Women's and Children's Hospital (WCH) in SA, and Perth Children's Hospital (PCH) in WA. The $\mathrm{WCH}$ and $\mathrm{PCH}$ are the sole referral sites for children with cancer for their states, with WCH also serving as the primary referral centre for children with suspected malignancies from the NT.

Cases were ascertained from hospital-based registries at WCH and PCH. Demographic characteristics, Indigenous status (self-reported) and diagnostic features at presentation, including full blood count, biochemistry, peripheral blast count, blast immunophenotype, blast genotype, molecular studies, central nervous system status, final risk stratification, treatment regimen and outcome were collected from medical records. The World Health Organisation classification of myeloid neoplasms and acute leukaemia was used for classification into diagnostic categories ${ }^{10}$. Final risk stratification was according to the co-operative group treatment protocols used in the respective centres, i.e. primarily the Children's Oncology Group for WA and the Berlin-Frankfurt-Munster protocols for SA/NT.

Residential address at diagnosis was categorised, first, by geographic remoteness using the Australian Statistical Geography Standard Remoteness Index ('remote/very remote', 'outer regional,' 'inner regional' or 'major city') ${ }^{11}$ and, second, by area-based socioeconomic status (SES) using the Index of Relative Socioeconomic Disadvantage ('least disadvantaged' (quintile 5 scores), 'middle SES' (quintiles 2 to 4) and 'most disadvantaged' (quintile 1) ${ }^{12}$. The latter (referred to as Socio-economic Index) is derived from national census data and incorporates income, educational attainment, employment and type of occupation.

Ethics Approval

Approval for the study was obtained from the South Australian and Western Australian Human Research Ethics Committees (HRECs) and the Aboriginal Human Research Ethics Committee (AHREC).

\section{Statistical Analysis}

Overall survival was calculated as time in months between diagnosis and last follow-up or death. Patients were considered as lost to follow-up when they had failed to attend scheduled appointments for more than one year. Five-year overall survival rates were estimated using the Kaplan-Meier method with differences assessed using the log-rank test. Analyses were performed using SPSS version 25 (IBM, Somers, NY, USA). $\mathrm{p}$ values less than 0.05 were considered statistically significant.

\section{RESULTS}

Between January $1^{\text {st }} 2009$ and December $31^{\text {st }} 2018$, a total of 455 patients were diagnosed with acute leukaemia at WCH $(n=202)$ and PCH $(n=253)$. Over half of the children were males $(57.6 \%, n=262 / 455)$. Patient age at diagnosis ranged from 1 day to 17 years and 10 months (median age 5.0 years) (Table 1). No significant difference was detected between patients from SA/NT and WA with regards to gender, Indigenous status, leukaemia diagnosis, cytogenetic group, relapse rate and mortality status.

Indigenous status was self-reported for all cases. A total of 29 Aboriginal children were diagnosed with leukaemia, 15 from SA/NT (7.4\%) and 14 from WA (5.5\%). There were no individuals who identified as Torres Strait Islander from either centre. Aboriginal children were significantly more likely to have a higher number of siblings (2.1 vs. $1.3, \mathrm{p}=0.009)$. They were significantly older at diagnosis compared to non-Aboriginal children (median age 8.1 vs. 4.9 years, $\mathrm{p}=0.01$ ).

Type of leukaemia 
The majority of patients had acute lymphoblastic leukaemia (ALL) $(79.8 \%$; $=363 / 455)$, most of which were B-ALL $(87.9 \%, 319 / 363)$, with the remainder having T-ALL $(12.1 \%, 44 / 363)$. Among patients with BALL, blast genotype revealed that the largest group had hyperdiploid ALL $(37.0 \% ; n=118 / 319)$, followed by ETV6-RUNX1 fusion $(17.9 \% ; \mathrm{n}=57 / 319)$ and normal karyotype $(12.9 \%$; $=41 / 319)$ (Supplementary Table 1). $20.4 \%(\mathrm{n}=65 / 319)$ displayed a variety of other cytogenetics abnormalities.

Among patients with acute myeloid leukaemia (AML), the largest subsets had either t(8;21) fusion $(13.4 \%$, $\mathrm{n}=11 / 82)$ or $\mathrm{t}(15 ; 17)$ fusion $(13.4 \%, \mathrm{n}=11 / 82)$ (Supplementary Table 1$)$. There were 10 patients with AML whose cytogenetics were unknown. There was an over-representation of AML patients among Aboriginal children compared to non-Aboriginal children in SA/NT $(60.0 \%, 9 / 15$ vs. $14.4 \%, 27 / 187, \mathrm{p}=0.001)$. This difference was not observed in WA $(14.3 \%, 2 / 14$ vs. $22.6 \%, 54 / 239, \mathrm{p}=0.46)$, although this analysis was limited by the small number of Aboriginal patients diagnosed with AML in WA. All of the Aboriginal patients with AML were from Very Remote areas.

\section{Geographic variation}

Aboriginal children were less likely to live in major cities than non-Aboriginal children $(41.4 \%$ vs. $72.3 \%$, $\mathrm{p}<0.001$ ) (Table 1, Fig. 1A). No Aboriginal children included in the entire study cohort lived in the least disadvantaged areas compared to $19.5 \%$ of non-Aboriginal children $(\mathrm{p}=0.0028)$ (Table 1$)$. There was a higher proportion of Aboriginal children from middle SES compared to non-Aboriginal children $(75.9 \%$ vs. $60.1 \%$, $\mathrm{p}=0.028)$. Rates of residence within the most disadvantaged areas were similar between the groups $(20.7 \%$ vs. $19.7 \%$ ) (Table 1).

\section{Treatment and follow-up}

Compared to non-Aboriginal children, Aboriginal children were less likely to be enrolled on clinical trials $(34.5 \%$ vs. $53.1 \%, \mathrm{p}=0.03)$ and were more likely to be lost to follow-up $(41.4 \%$ vs. $13.2 \%, \mathrm{p}<0.001)$ (Table 2 ). There was no evidence of a difference in loss to follow-up by area-based SES ( $\mathrm{p}=0.95)$; however, around a third $(31.2 \%)$ of children from remote and very remote localities were lost to follow-up compared to $12.8 \%$ from major cities $(\mathrm{p}=0.009)$ (Table 2$)$.

All Aboriginal patients with ALL had low MRD-based response compared to $78.8 \%$ of non-Aboriginal patients $(\mathrm{n}=16 / 16$ vs. $\mathrm{n}=241 / 306, \mathrm{p}=0.041)$, however these numbers were small (Table 1$)$.

\section{Survival}

Remoteness was identified as a significant survival factor for the entire cohort of patients with leukaemia ( $\mathrm{p}=0.004$, Fig. 1B). Five-year overall survival was $91.7 \%$ (95\% CI $=87.9 \%-94.3 \%)$ for children with ALL (Table 3), ranging from $92.2 \%$ for non-Aboriginal children to $82.4 \%$ for Aboriginal children $(\mathrm{p}=0.07)$. The 5-year overall survival was $69.8 \%(95 \% \mathrm{CI}=56.7-79.5 \%)$ for children with AML (Table 3). Unlike ALL, there was no trend for a difference in AML survival according to ethnicity $(\mathrm{p}=0.88)$. Analysis according to leukaemia type revealed a trend for inferior survival for children from remote and very remote localities for both ALL ( $\mathrm{p}=0.08)$ and AML ( $\mathrm{p}=0.08)$ (Table 3$)$. Since a larger population of Aboriginal patients come from remote areas, there is an overlap between remoteness and Aboriginal ethnicity. No significant differences in survival were found by either state/territory or SES for ALL or AML (Table 3).

\section{DISCUSSION}

Our study provides evidence pointing to inequity in outcome for Aboriginal children and for children from remote and very remote localities with acute leukaemia in Australia. There was no association found between MRD-based response and either ethnicity or remoteness of residence, suggesting the lower survival among Aboriginal children and those from remote areas is independent of this measure.

It is widely known that cancer is more likely to be fatal in Indigenous adults compared to non-Indigenous counterparts $^{3}$. There have been few studies performed in Indigenous children, particularly within a specific cancer type. A study by Valery et al. revealed a reduced 5-year survival of $75 \%$ for Indigenous children with any type of cancer in Australia compared to $82.3 \%$ for non-Indigenous children ${ }^{3}$. Furthermore, Indigenous 
children had 1.36 times greater risk of death within 5 years of diagnosis after adjustments for rurality of residence, SES, cancer diagnosis and year of diagnosis ${ }^{3,13}$.

Previous studies worldwide have shown higher overall cancer incidence and mortality in minority ethnic groups, such as Maori and Pacific Islander people in New Zealand (NZ) ${ }^{4,5}$. Black children with acute leukaemia and those from other ethnic or racial minority groups in the United States of America have inferior survival compared to Caucasian children $^{6-8}$. Measures have been taken to address the inequalities in cancer outcome and incidence between Maori and non-Maori people, such as introducing specific services for Maori people ${ }^{4}$. Similar measures are required to address the gap in survival rates for Aboriginal children with leukaemia in Australia.

It has been estimated that $76 \%$ of Indigenous children under 15 years of age in NT reside in remote and very remote parts of Australia compared to $13 \%$ in SA and $36 \%$ in $\mathrm{WA}^{14}$. However, data on childhood cancer incidence by remoteness of residence and SES are scarce ${ }^{15}$. Consistent with previous studies ${ }^{2}$, our study has shown Aboriginal children were less likely to live in major cities. Applying the ARIA remoteness index, this showed that Aboriginal children were more likely to live in communities further away from medical centres (Table 1). Furthermore, no Aboriginal children in our study lived in the least disadvantaged areas. Whilst these numbers were small, this trend warrants further research.

A study by Youlden et al. revealed that children living in more urban areas in Australia had higher cancer rates overall, particularly for leukaemia and lymphoma ${ }^{15}$. It was postulated that the reduced incidence of childhood cancers in remote and very remote areas could potentially be related to reduced incidence of cancer in Indigenous residents or missed diagnosis due to non-presentation. The incidence rates of childhood cancer were slightly higher among children in the least socioeconomically disadvantaged areas in Australia compared to those in the most disadvantaged areas ${ }^{15}$. Whilst this study did not yield population data for SA, NT and WA, analysing rates of leukaemia between areas of remoteness and different SES should be considered for future studies.

Worldwide, there has been inconclusive evidence that geographic residence impacts on overall survival ${ }^{9}$ although previous Australian studies have indicated poorer rates of survival for children with cancer who live in more isolated parts ${ }^{9}$. One would expect that with increasing remoteness comes reduced access to healthcare and increased challenges associated with diagnosis, treatment and long-term follow-up ${ }^{15}$. It is not known whether the reduced survival in remote areas is due to the larger relative percentage of Indigenous people $^{9}$.

Generally, an inferior outcome has been observed in children with cancer from lower or middle-income countries; however, results from low socioeconomic demographics in high-income countries are less consistent ${ }^{16}$. A study from Queensland in the early 1980s reported a significant difference in incidence and survival among children with ALL across different SES, despite similar treatments being received ${ }^{9,17}$. This trend was also observed in Maori and Pacific people in $\mathrm{NZ}^{5}$. Further research is clearly warranted to evaluate causes of poorer outcomes in children from remote areas, lower socioeconomic advantage and from various ethnicities.

An overrepresentation of AML patients in Aboriginal children diagnosed and treated in SA (WCH) between 1978 and 1998 was first reported in an audit by Bartle et al. in $1999^{1}$. These patients were less likely to speak English and more likely to come from traditional Aboriginal communities ${ }^{1}$. This was confirmed 12 years later by Rotte et al. , who again found relatively more cases of AML diagnosed among Aboriginal children at WCH between 1997 and $2011^{2}$. The latest cohort of patients from the same geographical area (SA/NT) reported here has also shown a higher proportion of AML cases and again without obvious morphological phenotype or cytogenetic abnormality ${ }^{1}$. The over-representation of AML in Aboriginal children in SA/NT observed in three successive cohorts since $1978^{1-2}$ warrants further investigation and interestingly was not observed here in Aboriginal children from WA. Our study also seems to indicate lower overall survival of children with AML compared to current Australian Childhood Cancer Statistics $(69.8 \% \text { vs. } 77.6 \%)^{18}$, although this may be because the latter only includes children diagnosed before the age of 15 rather than 18 .

Despite small patient numbers, there was a significant difference observed in clinical trial enrolment and 
follow-up among Aboriginal children. Previous studies have reported lower participation rates in clinical trials $^{2}$, which is likely to contribute to a less optimal outcome given less rigorous adherence to diagnostic and therapeutic guidelines ${ }^{2}$. Cultural and language barriers can also make discussions regarding randomised trial and informed consent challenging ${ }^{2}$.

Consistent with previous studies ${ }^{2}$, we found a lower rate of follow-up among Aboriginal children, with a statistically significant increase in those lost to follow-up. As discussed previously, this may reflect inequality in healthcare access for those in rural and remote communities. Improvements could be made by expediting use of tele-conferencing to support a shared care model and utilisation of rural outreach oncology nurses to enhance communication with local healthcare workers ${ }^{2}$. Furthermore, simplification of care, community involvement, peer support and decentralisation of care may also improve service delivery for Aboriginal and remote people ${ }^{19}$.

Strengths from this study are the high level of data quality obtained from the contributing centres. Limitations include the relatively small cohort size and subsequently small number of Aboriginal children included, leading to reduced statistical power. Furthermore, given only patients were included from SA, NT and WA, there is not complete population coverage of data. This could potentially introduce bias, however this risk is small given most children diagnosed with leukaemia in these states/territories are referred to the major paediatric oncology treating hospitals included in the study. Indigenous status was defined by self-assessment at the involved centres and the accuracy of these data is unknown. Nevertheless, any misclassification of Indigenous status is likely to be random with respect to the factors examined here, thus is unlikely to have biased our results.

\section{CONCLUSION}

Aboriginal children with acute leukaemia have a trend for increased mortality, are less likely to be enrolled onto clinical trials and are more likely to be lost to follow-up. Geographic remoteness of residence was identified as an adverse prognostic factor for all children with leukaemia. Additional strategies are required to ensure children from regional/rural locations and Aboriginal children with leukaemia receive appropriate service delivery and resource allocation to improve their survival and follow-up.

\section{CONFLICTS OF INTEREST: None}

ACKNOWLEDGEMENTS: Not applicable

\section{REFERENCES:}

1. Bartle S, Rice M. Overrepresentation of aboriginal children in acute myeloid leukaemia: the experience of one institution. Journal of Paediatrics and child health 2000;36(3).

2. Rotte L, Hansford J, Kirby M, Osborn M, Suppiah R, Ritchie P, Tapp H, Rice M, Revesz T. Cancer in Australian Aboriginal children: Room for Improvement. Journal of Paediatrics and Child Health2012:49(1).

3. Valery P, Youlden D, Baade P, Ward L, Green A, Aitken J. Cancer survival in Indigenous and nonIndigenous Australian children: what is the difference? Cancer Causes Control 2013:24:2099-2016.

4. Dachs G, Currie M, McKenzie F, Jeffreys M, Cox B, Foliaki S, le Marchand L, Robinson B. Cancer disparities in Indigenous Polynesian populations: Maori, Native Hawaiians, and Pacific People. The Lancet 2008:9:473-484.

5. Douglas N, Dockerty J. Survival by ethnicity for children diagnosed with cancer in New Zealand during 1990-1993. Journal of Paediatrics and Child Health 2007: 43:173-177.

6. Kadan-Lottick N, Ness K, Bhatia S, Gurney J. Survival variability by race and ethnicity in childhood acute lymphoblastic leukaemia.JAMA 2003:290:2008-2014

7. Ries L, Smith M, Gurney J et al. Cancer incidence and Survival Among Children and Adolescents: United States SEER Program 1975-1995. Bethesda, MD. National Cancer Institute, 1999, SEER Program, NIH Pub. No. 99-4649. 
8. Bhatia S, Sather H, Heerema N, Trigg M, Gaynon P, Robison L. Racial and ethnic differences in survival of children with acute lymphoblastic leukaemia. Blood 2002:100:1957-1964.

9. Youlden D, Baade P, Valery P, Ward L, Green A, Aitken J. Differentials in Survival for Childhood Cancer in Australia by Remoteness of Residence and Area Disadvantage. Cancer Epidemiol Biomarkers Prev 2011:20(8):1649-1656.

10. Swerdlow S, Campo E, Harris N, Jaffe E, Pileri S, Stein H, Thiele J. World Health Organisation Classification of Tumours. WHO Classification of Tumours of Haematopoietic and Lymphoid Tissues. Revised $4^{\text {th }}$ Edition. Lyon. 2017:10-12.

11. Australian Bureau of Statistics. Australian Statistical Geography Standard (ASGS): Volume 5 - Remoteness Structure, July 2016. Cat No. 1270.0.55.005. Canberra, Australia: ABS; 2018.

12. Australian Bureau of Statistics. Census of Population and Housing: Socio-Economic Indexes for Areas (SEIFA), Australia, 2016. Cat No. 2033.0.55.001. Canberra, Australia: ABS; 2018.

13. Valery P, McBride C. Sustainable care for indigenous children with cancer. The Lancet Oncology 2020:21. 489-491.

14. Australian Bureau of Statistics. Estimates of Aboriginal and Torres Strait Islander Australians, June 2016. Cat No. 3238.0.55.001. Canberra, Australia: ABS; 2018.

15. Youlden D, Baade P, Valery P, Hassal T, Ward L, Green A, Aitken J. Area-based Differentials in Childhood Cancer Incidence in Australia, 1996-2006. Pediatric Blood and Cancer 2012:58:390-394.

16. Gupta S, Wilejto M, Pole J, Guttmann A, Sung L. Low Socioeconomic Status Is Associated with Worse Survival in Children with Cancer: A Systematic Review. Socioeconomic Status and Childhood Cancer 2014:9(2):1-13.

17. Youlden D, Aitken J. Childhood cancer in Australia, 1983-2015. Brisbane: Cancer Council Queensland. 2019.

18. Australian Childhood Cancer Registry, Cancer Council Queensland. Australian Childhood Cancer Statistics Online. CCQ: Brisbane, Australia; 2018. Based on data from the ACCR (1983-2015).

19. Atun R, Bhakta N, Denburg A, Frazier L, Friedrich L, Friedrich P, et al. Sustainable care for children with cancer: a Lancet Oncology Commission. The Lancet 2020:21. 185-224.

Table 1 Patient Characteristics. Percentages next to numbers are within the cohort, percentages under the numbers are within the variable (column). Columns and rows don't always add up to $100 \%$ where source data are missing. CNS status; CNS1: absence of blast cells in CSF; CNS2: $<5 / \mu \mathrm{l}$ WBCs and cytospin positive for blasts; CNS3: $>5 / \mu \mathrm{l}$ WBCs and cytospin positive for blasts and/or clinical signs of CNS leukaemia.

Table 2 Patient Follow-Up Status. Percentages next to numbers are within the cohort, percentages under the numbers are within the variable (column). Columns and rows don't always add up to $100 \%$ where source data are missing.

Table 3 Five-year overall survival by patient characteristics. Note: Survival analysis excludes patients where mortality status at last follow-up date was unknown.

Figure 1A Remoteness Distribution by State and Ethnicity. Bar graph of percentage patients living in areas of remoteness between centres. WA $=$ Western Australia; $\mathrm{SA}=$ South Australia; NT $=$ Northern Territory. Overall $\mathrm{p}<0.001$.

Figure 1B Overall Survival according to Remoteness of home. Univariate Kaplan-Meier survival curve by area of remoteness.

\section{Hosted file}

Table 1.docx available at https://authorea.com/users/356375/articles/479260-outcome-foraustralian-children-with-acute-leukaemia-is-influenced-by-ethnicity-and-the-metroregional-divide

\section{Hosted file}

Table 2.docx available at https://authorea.com/users/356375/articles/479260-outcome-for- 
australian-children-with-acute-leukaemia-is-influenced-by-ethnicity-and-the-metroregional-divide

\section{Hosted file}

Table 3.docx available at https://authorea.com/users/356375/articles/479260-outcome-foraustralian-children-with-acute-leukaemia-is-influenced-by-ethnicity-and-the-metroregional-divide

A

Remoteness and Ethnicity

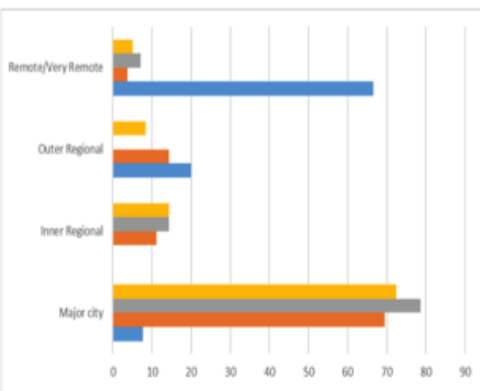

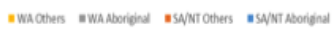

Cumulative Survival according to Remoteness of home

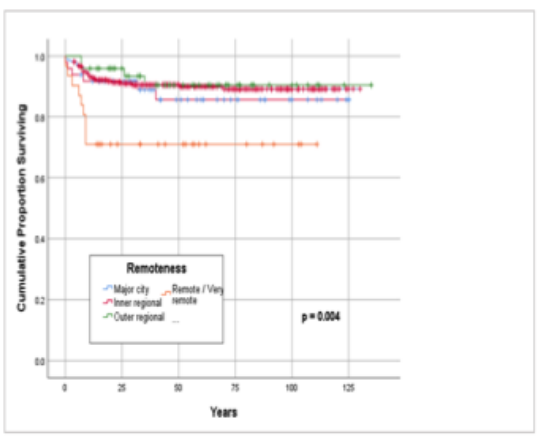

Remoteness by State and Ethnichy. Bar graph of percentage patients living in areas of remoteness between centres. WA = Western Australia; $\mathrm{SA}=$ South Australia; NT = Northern Territory. 